\title{
Multi-Operator Spectrum Sharing Models under Different Cooperation Schemes for Next Generation Cellular Networks
}

\author{
Mohammad N. Patwary ${ }^{*}$, Raouf Abozariba ${ }^{\dagger}$ and Md Asaduzzaman ${ }^{\dagger}$ \\ *School of Computing and Digital Technology, \\ Birmingham City University, Birmingham B4 7BD, UK \\ ${ }^{\dagger}$ School of Creative Arts and Engineering, \\ Staffordshire University, Staffordshire ST4 2DE, UK \\ mohammad.patwary@bcu.ac.uk, \{r.abozariba $\},\{$ md.asaduzzaman $\} @$ staffs.ac.uk
}

\begin{abstract}
Spectrum sharing between operators with exclusive licensing have become a major concern for mobile network operators and regulators to respond to the growing spectrum demand of the multimedia applications. One of the important issues in spectrum sharing is to determine the potential benefit when multi-operators share the resources under certain mutual agreements. The paper focuses on dynamic spectrum sharing management in next generation cellular networks. We propose three loss network models and derive the closed form expression of blocking probability, each having specific level of cooperation and interaction. The analytical frameworks are presented to analyze the benefits due to multi-operator cooperation for spectrum sharing. This quantifies the operators' gains and degradations of operators engaged in cooperative arrangements. We also analyze the overall network performance in terms of spectrum utilization and present a detailed comparisons between the proposed analytical frameworks.
\end{abstract}

Index Terms-Spectrum sharing, spectrum allocation, blocking probability, network utilization.

\section{INTRODUCTION}

The current static allocation of spectrum blocks allocated to specific purposes and to certain operators has led to serious implications [1], [2]. For example, large part of the allocated spectrum to military, government and public safety is underutilised. On the other hand the cellular frequencies are well utilized but due traffic misbalance, the utilization varies between license holders over time and space [3]. Thus, regulatory bodies in Europe and North America aim to improve the spectrum utilisation by liberalising part of the statically allocated spectrum [4], [5]. Such a rule has driven many researchers to design and analyze the effectiveness of spectrum sharing under various settings and conditions. The aim of spectrum sharing in cellular networks is to take advantage of the fluctuations of demand of several coexisting operators for an opportunistic allocation of unused spectrum resources. Primarily, for the purpose of minimizing the call congestion as well as increasing the over all spectra utilization [6].

In the recent years, a number of research papers focused on analyzing systems' performance in terms of blocking probability and network wide spectrum utilization. Modeling of capacity management for cellular networks using Poisson process is presented in literature. In [7], multi-class service scenario is modeled using multi-dimensional Markov Chain. The Markov chain is further approximated using the Erlang approximation method to evaluate the activity factor of a base station. The work in [8] presents the analytical expressions for blocking probability to evaluate the performance of the wireless network virtualization under different sharing policies. The analytical results confirm that the framework is accurate and showing its suitability to serve as a tool to design an efficient policy for sharing the physical spectrum in the wireless network virtualization. Blocking probability assessment when both secondary user traffic and primary user traffic are present in the system have been investigated in [9]. The results obtained are validated through live mobile data of primary user network. The authors in [10] presents an analytical formulation of the dynamic spectrum allocation problem for handling multi-class services in two cellular radio systems using a complete sharing (CS) scheme. In [11], multi-dimensional Markov process is used to obtain results on the blocking probabilities. In [12], authors study the system performance using two-dimensional Markov chain with handover and new calls based on the Erlang B systems. In [13], the authors studied cooperative resource sharing for wireless communication networks. In particular, the authors studied four models and present the analytical results of blocking probability for each model.

The majority of the aforementioned studies consider limited level of cooperation and only two operators in the network, therefore, it is easier to analyze the system performance. In this paper, in addition to the previous works, we consider the scenario emerging from spectrum sharing where one secondary operator interacts with multi-primary operators according to a defined mutual agreements. We analyze three types of multioperator joint spectrum management schemes by considering a loss system. Analysis and modeling of loss system are vital for the ubiquitous real-time multimedia (voice and video) communications where delay is not tolerable. The modeling and analysis of loss systems are increasingly important due to the growing percentage of the multimedia traffic.

The contribution of this paper is in quantifying the grade of service (GoS) improvements of operators when they 
engage in spectrum sharing schemes. Depending on the amount of the traffic in a cooperative setting, the blocking probability is calculated for each operator individually. The derived formulae take into account the arrival rate, service rate, traffic intensity and the available capacity of all operators involved in the cooperation. We also model the mutual agreement structures for each proposed model by incorporating the interactions into our proposed mathematical models. Three levels of cooperations are considered: 1) uni-directional cooperation 2) bi-directional cooperation and 3) bi-directional cooperation with pooled capacity. All these cases are modeled precisely and quantified by their individual operators' blocking probabilities. In addition, we derive a common performance comparison framework to evaluate the proposed models by calculating the spectrum utilization of the formed agreements. Analytical results are provided to demonstrate the accuracy of blocking probability calculations.

The rest of the paper is organized as follows. The system model is described in the next Section. Section III presents the spectrum sharing models and describes our mathematical approach. In Section IV, we present our findings. Finally, Section V summarizes our conclusions.

\section{SySTEM MODEL}

We consider a network consisting of four operators. An operator could be a primary operator, secondary operator or both, depending on the chosen arrangement between operators, see Figures 1, 2 and 3. We first assume that each of the operators in the network owns spectrum band which is orthogonally divided into $c_{i} \in \mathbb{Z}^{+}$resource blocks. Each operator serve users with Poisson distributed arrivals and mean rate $\lambda_{i}$ and the service rate $\mu_{i}$. In a non-sharing model, each operator in the network would operate independently and the blocking probability in this case can be easily calculated using an Erlang system giving $E\left(c_{i}, \lambda_{i}, \mu_{i}\right)$ [14], [13]. However in a cooperative network if one or more operators are underloaded then it may allow other operators with high traffic to use their under-utilized resources under a mutual agreement.

A first-come-first-served scheduling system is considered to allow stability and eliminate channel interference. If a channel is being used by an operator then primary operator waits until a channel is vacated by its current occupier. Channel requests are granted completely, in which fragmentation is not modeled.

In the system where multi-operators covering the same geographical area, the SNO aims to find the operators with available channels in order to balance the load across all available resources without causing one operator to be overloaded while other operators are in an underloaded state. Such a set up will ensure better utilization of spectrum as we will see later in Section IV. The PNOs who experiences a drop in the average arrival rate $\lambda_{i}$ will be preferable to the SNO. Similarly, PNOs who is experiencing an increase of channel request rates would not be accessible by the SNO. When all PNOs channels are busy then the SNO will have to drop the new arrival channel requests. In this paper we consider a non-adjustable service rate to provide a standardized service quality.

Operators benefit from temporal variation in the traffic by allowing each other to use their idle channels with mutual agreements. We discuss three possible models in cellular networks. Uni-Directional cooperation; Bi-Directional cooperation; Bi-Directional cooperation with emergency capacity. The models are discussed in details in the Section III

\section{DYNAMIC SPECTRUM SHARING MODELS}

In this section, we develop models for dynamic spectrum sharing under different resource sharing schemes. Three models with complex sharing schemes are proposed which are described in the subsequent sections.

\section{A. Model A: Uni-Directional cooperation}

Consider a network with three primary and one secondary operators where the secondary operator aims to borrow spectrum from the primary operators under a uni-directional leasing agreement as shown in Figure 1. Our main objective is to find the impact of the secondary operator on the grade of service and spectrum utilization. We assume that the channel requests follow Poisson processes with arrival rates $\lambda_{i}, i=1,2,3$ for $i$ th PNO and $\lambda_{0}$ for the SNO and exponential channel holding time with rates $\mu_{i}, i=1,2,3$ for $i$ th PNO and $\mu_{0}$ for the SNO. The offered load for the $i$ th operator is then defined as $\rho_{i}=\lambda_{i} / \mu_{i}$. Denote the capacity of the $i$ th operator as $c_{i}, i=0,1,2,3$.

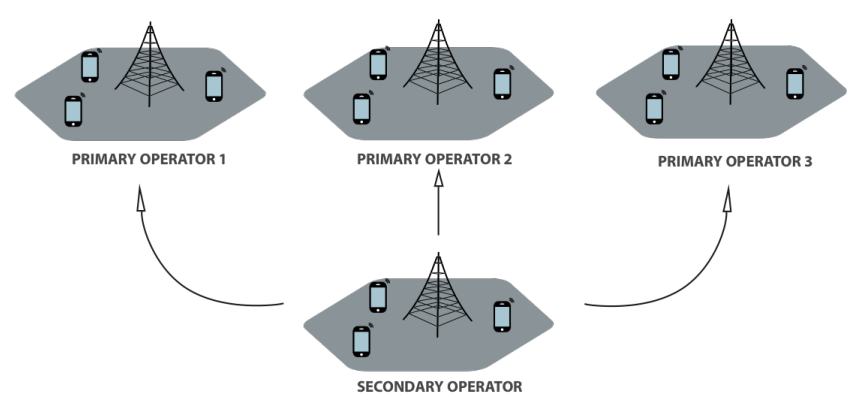

Fig. 1: Uni-Directional service operators sharing network

Let $X_{i}(t), i=1,2,3$ be the number of channels in $i$ th primary network operator (PNO), $X_{0}(t)$ be the number of channels in secondary network operator (SNO) and $X_{0 i}(t), i=$ $1,2,3$ be the number of channels borrowed by the SNO from the $i$ th PNO. Then a state of the process is a vector defined by $\mathbf{X}=\left(X_{0}(t), X_{1}(t), X_{2}(t), X_{3}(t), X_{01}(t), X_{02}(t), X_{03}(t)\right)$ which is a Markov chain with state space

$$
\begin{aligned}
& \Omega=\left\{\left(n_{0}, n_{1}, n_{2}, n_{3}, n_{01}, n_{02}, n_{03}\right)\right.: n_{0} \leq c_{0}, \\
&\left.n_{i}+n_{0 i} \leq c_{i}, i=1,2,3\right\}
\end{aligned}
$$

The transition rates of the process are defined by (2). 


$$
q\left(\mathbf{n}, \mathbf{n}^{\prime}\right)= \begin{cases}\lambda_{0}(t) & \mathbf{n}^{\prime}=\mathbf{n}+\mathbf{e}_{0} \text { or } \mathbf{n}^{\prime}=\mathbf{n}+\mathbf{e}_{01} \text { if } n_{0}=c_{0} \cap n_{1}+n_{01}<c_{1} \\ & \text { or } \mathbf{n}^{\prime}=\mathbf{n}+\mathbf{e}_{02} \text { if } n_{0}=c_{0} \cap n_{1}+n_{01}=c_{1} \cap n_{2}+n_{02}<c_{2} \\ & \text { or } \mathbf{n}^{\prime}=\mathbf{n}+\mathbf{e}_{01} \text { if } n_{0}=c_{0} \cap n_{1}+n_{01}=c_{1} \cap n_{2}+n_{02}=c_{2} \cap n_{3}+n_{03}<c_{3} \\ \lambda_{i}(t) & \mathbf{n}^{\prime}=\mathbf{n}+\mathbf{e}_{i}, i=1,2,3 \\ n_{i} \mu_{i}(t) & \mathbf{n}^{\prime}=\mathbf{n}-\mathbf{e}_{i}, i=0,1,2,3 \\ n_{0 i} \mu_{i}(t) & \mathbf{n}^{\prime}=\mathbf{n}-\mathbf{e}_{0 i}, i=1,2,3\end{cases}
$$

where $\mathbf{e}_{0}$ and $\mathbf{e}_{0 i}$ are unit vectors.

$$
\begin{aligned}
\frac{\mathrm{d} \pi(\mathbf{n}, t)}{\mathrm{d} t}= & {\left[\lambda_{0}(t) \cdot\left(\mathbb{1}\left(n_{0}<c_{0}\right)+\mathbb{1}\left(n_{0}=c_{0} \cap_{i \in\{1,2,3\}} \mathbf{n}+\mathbf{e}_{0 i}\right)\right)+\sum_{i=1}^{3} \lambda_{i}(t) \cdot \mathbb{1}\left(n_{i}+n_{0 i}<c_{i}\right)\right] \cdot \pi\left(\left(\mathbf{n}-\mathbf{e}_{i}\right), t\right) } \\
& +\sum_{i=0}^{3}\left(n_{i}+1\right) \mu_{i}(t) \pi\left(\left(\mathbf{n}+\mathbf{e}_{i}\right), t\right)+\sum_{i=1}^{3}\left(n_{0 i}+1\right) \mu_{i 0}(t) \cdot \pi\left(\mathbf{n}+\mathbf{e}_{0 i}\right)-\left[\lambda_{0}(t) \cdot\left(\mathbb{1}\left(n_{0}<c_{0}\right)+\mathbb{1}\left(n_{0}=c_{0} \cap_{i \in\{1,2,3\}} \mathbf{n}+\mathbf{e}_{0 i}\right)\right)\right. \\
& \left.\left.+\sum_{i=1}^{3} \lambda_{i}(t) \cdot \mathbb{1}\left(n_{i}+n_{0 i}<c_{i}\right)+\sum_{i=0}^{3} n_{i} \mu_{i}(t)+\sum_{i=1}^{3} n_{0 i} \mu_{i}(t)\right)\right] \pi(\mathbf{n}, t)
\end{aligned}
$$

Denote the steady state distribution by $\pi(\mathbf{n}, t)$ which can be obtained by solving the Kolmogorov forward equation given by (3).

Solving the Kolmogorov forward equations (3) by equating at 0 , we obtain the closed form solution of the equilibrium distribution which is

$$
\pi(\mathbf{n})=\mathcal{G}^{-1} \frac{\rho_{0}^{\left(n_{0}+n_{01}+n_{02}+n_{03}\right)}}{\left(n_{0}+n_{01}+n_{02}+n_{03}\right) !} \cdot \frac{\rho_{1}^{n_{1}} \rho_{2}^{n_{2}} \rho_{3}^{n_{3}}}{n_{1} ! n_{2} ! n_{3} !}, \quad \forall \mathbf{n} \in \Omega
$$

where

$$
\mathcal{G}=\sum_{\mathbf{n} \in \Omega}\left[\frac{\rho_{0}^{\left(n_{0}+n_{01}+n_{02}+n_{03}\right)}}{\left(n_{0}+n_{01}+n_{02}+n_{03}\right) !} \cdot \frac{\rho_{1}^{n_{1}} \rho_{2}^{n_{2}} \rho_{3}^{n_{3}}}{n_{1} ! n_{2} ! n_{3} !}\right] .
$$

One of the main goals of deriving the equilibrium distribution is to calculate the blocking probability or call congestion rate. The formula for blocking probability can be derived from the closed-form solution (4). The blocking probability for an operator $i, i=0,1,2,3$ is then given by

$$
\begin{aligned}
P_{b_{i}}(t)= & \sum_{\mathbf{n} \in S_{R}} \pi(\mathbf{n}, t) \\
= & \frac{\sum_{\mathbf{n} \in S_{R}} \frac{\rho_{0}^{\left(n_{0}+n_{01}+n_{02}+n_{03}\right)}}{\left(n_{0}+n_{01}+n_{02}+n_{03}\right) !} \cdot \frac{\rho_{1}^{n_{1}} \rho_{2}^{n_{2}} \rho_{3}^{n_{3}}}{n_{1} ! n_{2} ! n_{3} !}}{\sum_{\mathbf{n} \in \Omega} \frac{\rho_{0}^{\left(n_{0}+n_{01}+n_{02}+n_{03}\right)}}{\left(n_{0}+n_{01}+n_{02}+n_{03}\right) !} \cdot \frac{\rho_{1}^{n_{1}} \rho_{2}^{n_{2}} \rho_{3}^{n_{3}}}{n_{1} ! n_{2} ! n_{3} !}} \forall \mathbf{n} \in \Omega
\end{aligned}
$$

where the set $S_{R}$ is the restricted state space, and varies for the SNO and PNOs. For the SNO, it is defined as

$$
\begin{aligned}
S_{R}=\left\{\mathbf{n} \in \Omega \mid\left(n_{0}=c_{0} \cap n_{01}+n_{11}\right.\right. & =c_{1} \cap n_{02}+n_{22}=c_{2} \\
& \left.\left.\cap n_{03}+n_{33}=c_{3}\right)\right\},
\end{aligned}
$$

and for the $i$ th PNO, $S_{R}$ can be replaced by $S_{i}$ and defined as

$$
S_{i}=\left\{\mathbf{n} \in \Omega \mid\left(n_{i}+n_{0 i}=c_{i}\right)\right\}, \quad i=1,2,3 .
$$

\section{B. Model B: Bi-Directional cooperation}

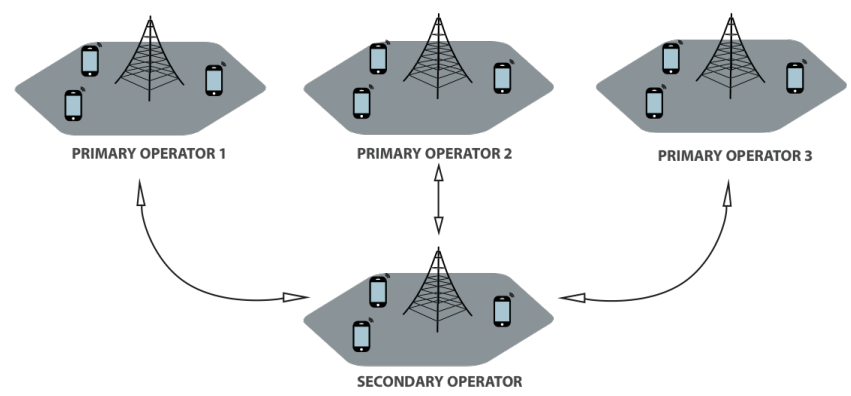

Fig. 2: Bi-Directional service operators sharing network

In bi-directional cooperative model, in addition to unidirectional operation primary operators are also allowed to borrow spectrum from the secondary operators when they require as shown in Figure 2.

Deriving Kolmogorov forward equation and solving we obtain the equilibrium probability distribution as given in 
equation (9).

$$
\begin{aligned}
\pi(\mathbf{n})=\mathcal{G}^{-1} & \frac{\rho_{0}^{\left(n_{0}+n_{01}+n_{02}+n_{03}\right)}}{\left(n_{0}+n_{01}+n_{02}+n_{03}\right) !} \\
& \cdot \frac{\rho_{1}^{\left(n_{1}+n_{10}\right)} \rho_{2}^{\left(n_{2}+n_{20}\right)} \rho_{3}^{\left(n_{3}+n_{30}\right)}}{\left(n_{1}+n_{10}\right) !\left(n_{2}+n_{20}\right) !\left(n_{3}+n_{30}\right) !}, \quad \forall \mathbf{n} \in \Omega
\end{aligned}
$$

where

$$
\begin{aligned}
\mathcal{G}=\sum_{\mathbf{n} \in \Omega}\left[\frac{\rho_{0}^{\left(n_{0}+n_{01}+n_{02}+n_{03}\right)}}{\left(n_{0}+n_{01}+n_{02}+n_{03}\right) !}\right. & \\
& \left.\cdot \frac{\rho_{1}^{\left(n_{1}+n_{10}\right)} \rho_{2}^{\left(n_{2}+n_{20}\right)} \rho_{3}^{\left(n_{3}+n_{30}\right)}}{\left(n_{1}+n_{10}\right) !\left(n_{2}+n_{20}\right) !\left(n_{3}+n_{30}\right) !}\right]
\end{aligned}
$$

The blocking probability formula for quantifying the GoS can be given by

$$
\begin{aligned}
P_{b_{i}}(t)= & \sum_{\mathbf{n} \in S_{R}} \pi(\mathbf{n}, t) \\
= & \frac{\sum_{\mathbf{n} \in S_{R}} \frac{\rho_{0}^{\left(n_{0}+n_{01}+n_{02}+n_{03}\right)}}{\left(n_{0}+n_{01}+n_{02}+n_{03}\right) !} \cdot \frac{\rho_{1}^{\left(n_{1}+n_{10}\right)} \rho_{2}^{\left(n_{2}+n_{20}\right)} \rho_{3}^{\left(n_{3}+n_{30}\right)}}{\left(n_{1}+n_{10}\right) !\left(n_{2}+n_{20}\right) !\left(n_{3}+n_{30}\right) !}}{\sum_{\mathbf{n} \in \Omega} \frac{\rho_{0}^{\left(n_{0}+n_{01}+n_{02}+n_{03}\right)}}{\left(n_{0}+n_{01}+n_{02}+n_{03}\right) !} \cdot \frac{\rho_{1}^{\left(n_{1}+n_{10}\right)} \rho_{2}^{\left(n_{2}+n_{20}\right)} \rho_{3}^{\left(n_{3}+n_{30}\right)}}{\left(n_{1}+n_{10}\right) !\left(n_{2}+n_{20}\right) !\left(n_{3}+n_{30}\right) !}} \forall \mathbf{n} \in \Omega
\end{aligned}
$$

where the set $S_{R}$ is the restricted state space for all operators.

For the SNO, it is defined as

$$
\begin{aligned}
S_{R}=\left\{\mathbf{n} \in \Omega \mid\left(n_{0}=c_{0} \cap n_{01}+n_{11}\right.\right. & =c_{1} \cap n_{02}+n_{22}=c_{2} \\
& \left.\left.\cap n_{03}+n_{33}=c_{3}\right)\right\},
\end{aligned}
$$

and for the $i$ th PNO, $S_{R}$ can be replaced by $S_{i}$ and defined as

$$
S_{i}=\left\{\mathbf{n} \in \Omega \mid n_{0}+n_{i 0}=c_{0} \cap\left(n_{i}+n_{0 i}=c_{i}\right)\right\}, \quad i=1,2,3 . \text { (13) }
$$

C. Model C: Bi-Directional cooperation with pooled resources

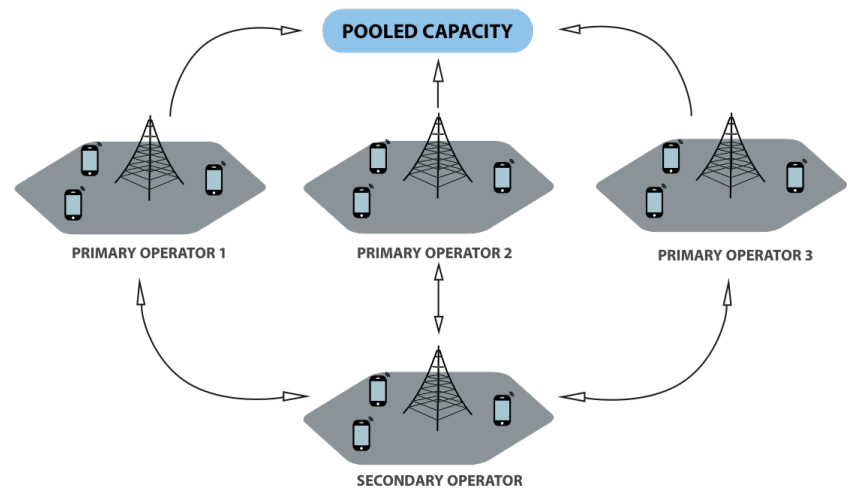

Fig. 3: Bi-Directional with pooled capacity service operators sharing network
The bi-directional cooperation with pooled resources model is similar to Model B with additional pooled resources denoted by $c_{p}$, which can be accessed by any of the PNOs under first-come-first-served discipline, see Figure 3. The pooled resources is considered as a last resort for the PNOs when the SNO's channels are also occupied.

$$
\begin{aligned}
& \pi(\mathbf{n})=\mathcal{G}^{-1} \frac{\rho_{0}^{\left(n_{0}+n_{01}+n_{02}+n_{03}\right)}}{\left(n_{0}+n_{01}+n_{02}+n_{03}\right) !} \\
& \frac{\rho_{1}^{\left(n_{1}+n_{10}+n_{1 p}\right)} \rho_{2}^{\left(n_{2}+n_{20}+n_{2 p}\right)} \rho_{3}^{\left(n_{3}+n_{30}+n_{3 p}\right)}}{\left(n_{1}+n_{10}+n_{1 p}\right) !\left(n_{2}+n_{20}+n_{2 p}\right) !\left(n_{3}+n_{30}+n_{3 p}\right) !} \\
& \forall \mathbf{n} \in \Omega \quad(14)
\end{aligned}
$$

where

$$
\begin{aligned}
\mathcal{G}= & \sum_{\mathbf{n} \in \Omega}\left[\frac{\rho_{0}^{\left(n_{0}+n_{01}+n_{02}+n_{03}\right)}}{\left(n_{0}+n_{01}+n_{02}+n_{03}\right) !}\right. \\
& \left.\cdot \frac{\rho_{1}^{\left(n_{1}+n_{10}+n_{1 p}\right)} \rho_{2}^{\left(n_{2}+n_{20}+n_{2 p}\right)} \rho_{3}^{\left(n_{3}+n_{30}+n_{3 p}\right)}}{\left(n_{1}+n_{10}+n_{1 p}\right) !\left(n_{2}+n_{20}+n_{2 p}\right) !\left(n_{3}+n_{30}+n_{3 p}\right) !}\right]
\end{aligned}
$$

The blocking probability formula for Model $\mathrm{C}$ can be given by

$$
\begin{aligned}
P_{b_{i}}(t)= & \sum_{\mathbf{n} \in S_{R}} \pi(\mathbf{n}, t) \\
= & \frac{\sum_{\mathbf{n} \in S_{R}} \frac{\rho_{0}^{\left(n_{0}+n_{01}+n_{02}+n_{03}\right)}}{\left(n_{0}+n_{01}+n_{02}+n_{03}\right) !} \cdot \frac{\rho_{1}^{\left(n_{1}+n_{10}+n_{1 p}\right)} \rho_{2}^{\left(n_{2}+n_{20}+n_{2 p}\right)} \rho_{3}^{\left(n_{3}+n_{30}+n_{3 p}\right)}}{\left(n_{1}+n_{10}+n_{1 p}\right) !\left(n_{2}+n_{20}+n_{2 p}\right) !\left(n_{3}+n_{30}+n_{3 p}\right) !}}{\sum_{\mathbf{n} \in \Omega}^{\left(n_{0}+n_{01}+n_{02}+n_{03}\right)}} \forall \mathbf{n} \in \Omega \\
& \frac{\rho_{1}^{\left(n_{1}+n_{10}+n_{1 p}\right)} \rho_{2}^{\left(n_{2}+n_{20}+n_{2 p}\right)} \rho_{3}^{\left(n_{3}+n_{30}+n_{3 p}\right)}}{\left(n_{0}+n_{01}+n_{02}+n_{03}\right) !} \cdot \frac{n_{10}}{\left(n_{1}+n_{10}+n_{1 p}\right) !\left(n_{2}+n_{20}+n_{2 p}\right) !\left(n_{3}+n_{30}+n_{3 p}\right) !}
\end{aligned}
$$


where the set $S_{R}$ is the restricted state space for all operators.

For the SNO, it is defined as

$$
\begin{array}{r}
S_{R}=\left\{\mathbf{n} \in \Omega \mid\left(n_{0}=c_{0} \cap n_{01}+n_{11}=c_{1} \cap n_{02}+n_{22}=c_{2}\right.\right. \\
\left.\left.\cap n_{03}+n_{33}=c_{3}\right)\right\},
\end{array}
$$

and for the $i$ th PNO, $S_{R}$ can be replaced by $S_{i}$ and defined as

$$
\begin{array}{r}
S_{i}=\left\{\mathbf{n} \in \Omega \mid n_{0}+n_{i 0}=c_{0} \cap\left(n_{i}+n_{0 i}=c_{i} \cap \sum_{i=1}^{3} n_{i p}=c_{p}\right)\right\}, \\
i=1,2,3 .
\end{array}
$$

\section{Marginal probability distribution and spectrum utilisation}

Spectrum utilization as the ratio of the average number of busy channels and the overall available number of channels in the network is an important parameter. As we aim to quantify the spectrum utilization we first calculate the marginal probability distribution of number of channels for each operator. The marginal probability distribution can be given by

$\pi\left(n_{i}\right)=\sum_{\mathbf{n} \in\left\{\Omega \backslash n_{i}\right\}} \pi(\mathbf{n})$

$\forall\left\{\begin{array}{l}i \in\{0,1,2,3,01,02,03\} \text { for Model A } \\ i \in\{0,1,2,3,01,02,03,10,20,30\} \text { for Model B } \\ i \in\{0,1,2,3,01,02,03,10,20,30,1 p, 2 p, 3 p\} \text { for Model C }\end{array}\right.$

Therefore, expected spectrum utilization of each model can be obtain as

$$
u\left(n_{i}\right)=\sum_{n_{i} \in \Omega} \frac{1}{c}\left[n_{i} \cdot \pi\left(n_{i}\right)\right] \quad \forall n_{i} \in \Omega
$$

where

$$
c=\left\{\begin{array}{l}
\left(c_{0}+c_{1}+c_{2}+c_{3}\right) \text { for Model A and B } \\
\left(c_{0}+c_{1}+c_{2}+c_{3}+c_{p}\right) \text { for Model } \mathrm{C}
\end{array}\right.
$$

\section{NuMERICAL RESUltS}

In this section, we show the impacts of system parameters to the models performance and verify our theoretical analysis presented in Section III.

\section{A. Effect of traffic intensity at secondary operator on blocking probability}

The first-come-first-served scheduling system, which we have used in our models, means that at saturation the primary operators have their bandwidth allocation reduced and hence we observe an increase in blocking probability, as shown in Figure 4. Below saturation point between an offered load of $\left(\rho_{0}=2: 3\right)$, the uni-directional cooperation outperform the bi-directional counterpart, see the zoomed part of Figure 4. However, when the network starts to reach saturation, the blocking probability of uni and bi-directional cooperation schemes are approximately equal and they increase exponentially as $\rho_{0} \rightarrow 10$. By deploying the bi-directional with pooled capacity model (Model $\mathrm{C}$ ), we notice that as the $\mathrm{X}$ axis of Figure 4 continues and with more traffic diverted to the primary operators' channels, the latter begin to rely on the pooled capacity (where $c_{p}=1$ ). This provides additional channels to the PNOs, which results in lower blocking probability compared to the first two models.

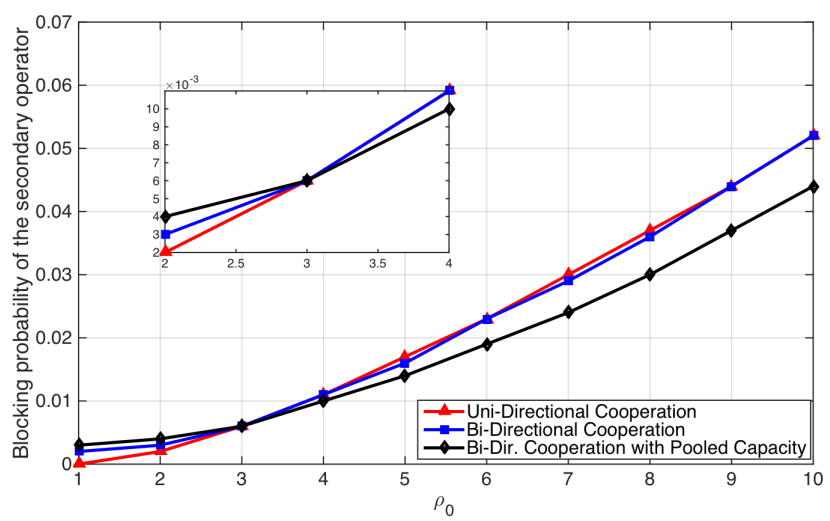

Fig. 4: Comparison of blocking probability for secondary operator using the proposed models with $\rho_{0}=1: 10$, see Table I for full configuration details.

B. Effect of traffic intensity at secondary operators on blocking probability

The blocking probability of the primary operators as a function of secondary operators traffic intensity is plotted in Figure 5a and 5b. From Figure 5a, we observe that a continuous increase in the blocking probability at operator 1 and 2 of new user requests as the traffic intensity of secondary operator increases while keeping the capacity of each operator constant. When the majority of the channels are occupied by respective licensed users, the primary operators use the pooled resources which is why we see Model C out perform Model A and $\mathrm{B}$ when the traffic is high. At low traffic Model B performs better well compared to Model A and C. In Figure $5 \mathrm{~b}$ the blocking probability of operator 3 is quantified using the three proposed models. From the figure, we find similar trends in blocking probability to Figure 5a with slight difference, which is caused by the variation in the parameters used in operator 2 and 3, as shown in the highlighted row of Table I.

\section{Effect of the number of available channels on blocking probability}

Figure 6 shows the blocking probability of secondary operator for each model when PNO 1 have different number of channels $\left(c_{1}=1: 10\right)$. In the network each operator has its own licensed channels, service rate and offered load as shown in the figure caption. This result indicates that Model A and B show similar performances of blocking probability. Model $\mathrm{C}$ in this case has the advantage due to higher number of channels. 


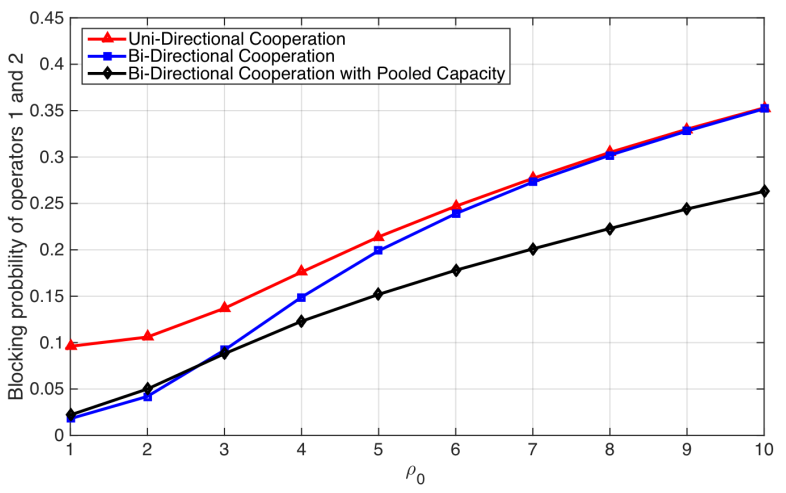

(a)

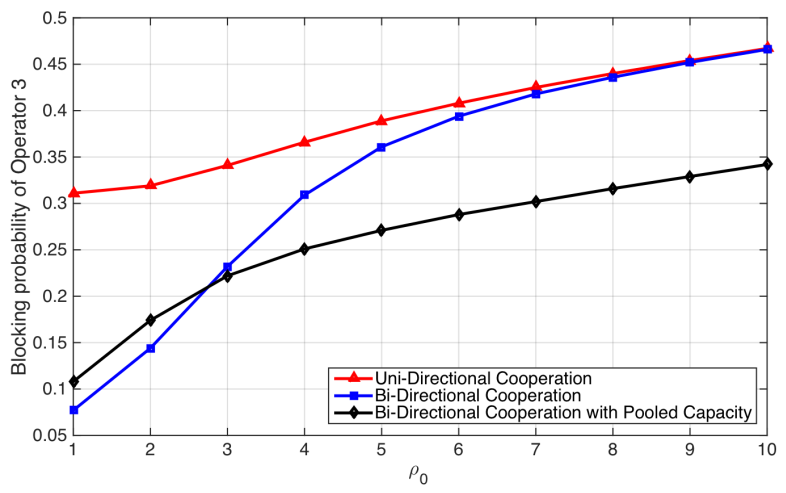

(b)

Fig. 5: Blocking probability for (a) operator 1 and 2 and (b) operator 3, see Table I for full configuration details.

TABLE I: Configurations used in Figure 4, 5a and $5 \mathrm{~b}$

\begin{tabular}{|c|c|c|c|c|c|c|c|c|c|}
\hline & \multicolumn{5}{|c|}{ Number of channels } & \multicolumn{4}{|c|}{ Traffic intensity } \\
\hline & SNO & PNO 1 & PNO 2 & PNO 3 & Pooled Capacity & SNO & PNO 1 & PNO 2 & PNO 3 \\
\hline Model A & 4 & 4 & 4 & 4 & - & $1: 10$ & 2 & 2 & 4 \\
\hline Model B & 4 & 4 & 4 & 4 & - & $1: 10$ & 2 & 2 & 4 \\
\hline Model C & 4 & 4 & 4 & 4 & 1 & $1: 10$ & 2 & 2 & 4 \\
\hline
\end{tabular}

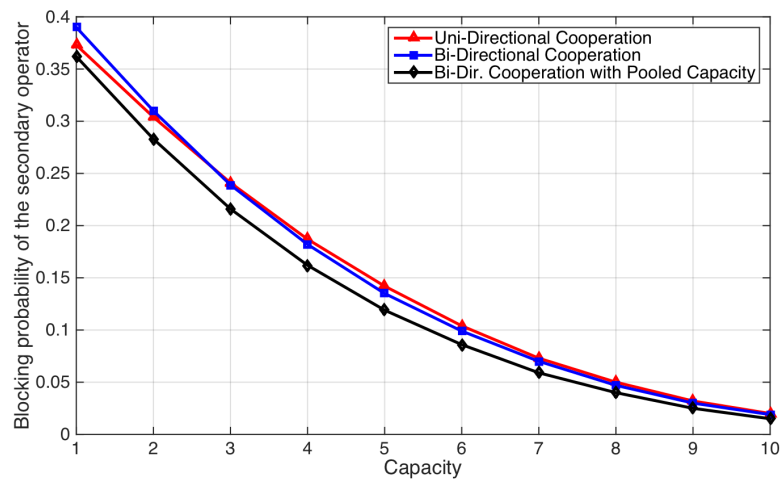

Fig. 6: Comparison of blocking probability for secondary operator with varying number of channels using the proposed models when $\rho_{s}=5, \rho_{1}=3, \rho_{2}=4, \rho_{3}=5, c_{0,2,3}=2, c_{1}=$ $1: 10, c_{p}=1$.

\section{Evaluation of spectrum utilization}

The performance measure discussed so far is concerned with the call congestion and focus on the performance of each individual operator. In this subsection we analyze the proposed model's efficiency in terms of spectrum utilization. We use the formulae derived in Section III-D and the simulation parameters shown in Table II. We show the change in the spectrum utilization against the traffic intensity at the SNO $\left(\rho_{0}=1: 10\right)$. In Figure 7 we can see that Model C is superior compared to the the other two models especially when $\rho_{0}<4$. We also notice that model $\mathrm{C}$ performance deteriorates when the traffic intensity is high $\rho_{0}>5$. When traffic load is less than 5 Model A and Model B provide similar performance due to increased saturation of channels. On average Model B performs best at $85 \%$ spectrum utilization with $2 \%$ higher than the uni-directional cooperation model and $0.5 \%$ higher than Model C.

We also investigate the spectrum utilization of the proposed models against the change in traffic intensity at the operators 1,2 and 3 , see Figure 8. Keeping $\rho_{0}$ fixed at 10 , we vary the traffic intensity of PNO 1, 2 and $3\left(\rho_{1}=\rho_{2}=\rho_{3}=5: 14\right)$. For a fair comparison, the total number of available channels is kept fixed for all Models as $\sum_{i} c_{i}=12 \forall i \in\{0,1,2,3\}$.

For traffic intensity below 6 the utilization of channels using Model A and B are equal. With excess offered load the difference between Model A and B becomes wider and considerably more for $\rho>13$. Under any offered load Model $\mathrm{C}$ shows the lowest level of efficiency. Considering traffic conditions occur at equal probability one could see that Model B provides the network with the highest spectrum utilization at $92.6 \%$.

\section{CONCLUSION}

Spectrum sharing in cellular networks have received much attention in recent years due to its efficiency of spectrum utilization and capability to improve the grade of service to subscribers. The efficiency is defined by spectrum utilization as the ratio of the average number of busy channels and the overall available number of channels in the network while the grade of service is defined by the blocking probability. In this paper we have presented three different models for dynamic spectrum sharing management in multi-operator cellular networks, operating with different spectrum holdings. Each 
TABLE II: Configurations used in Figure 7

\begin{tabular}{|c|c|c|c|c|c|c|c|c|c|}
\hline & \multicolumn{5}{|c|}{ Number of channels } & \multicolumn{4}{|c|}{ Traffic intensity } \\
\hline & $\mathrm{SNO}$ & PNO 1 & PNO 2 & PNO 3 & Pooled Capacity & SNO & PNO 1 & PNO 2 & PNO 3 \\
\hline Model A & 3 & 3 & 3 & 3 & - & $1: 10$ & 4 & 4 & 4 \\
\hline Model B & 3 & 3 & 3 & 3 & - & $1: 10$ & 4 & 4 & 4 \\
\hline Model C & 3 & 2 & 2 & 2 & 4 & $1: 10$ & 4 & 4 & 4 \\
\hline
\end{tabular}

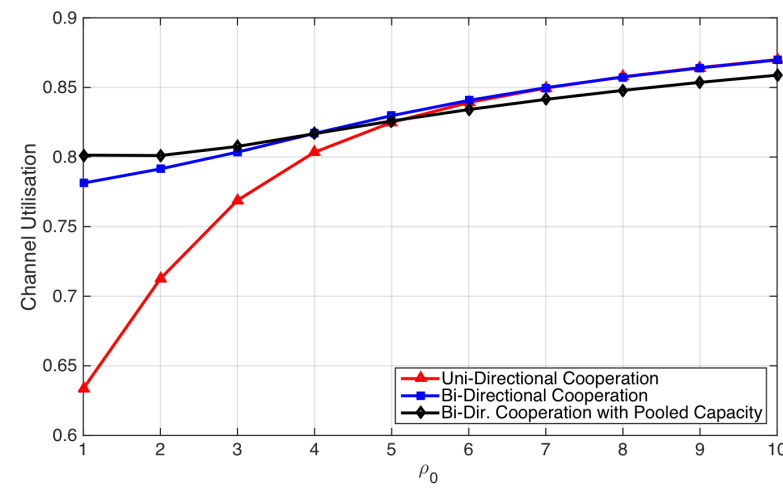

Fig. 7: Comparison of channel utilisation for secondary operator using the proposed models with $\rho_{0}=1: 10$.

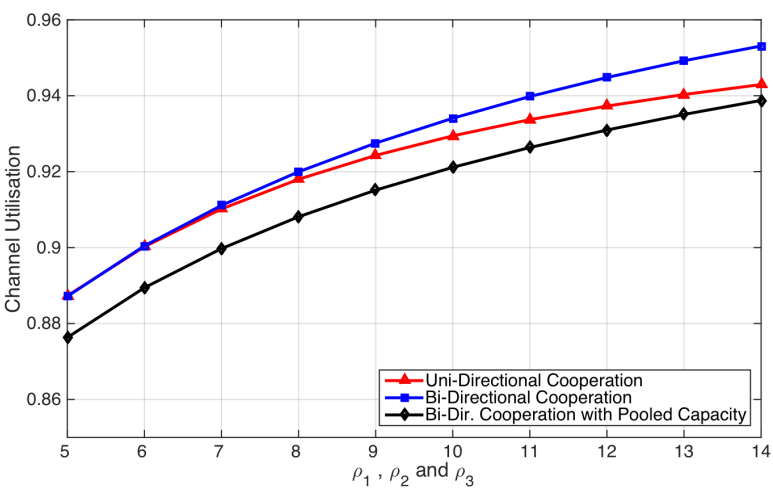

Fig. 8: Comparison of channel utilisation for secondary operator using the proposed models with $\rho_{1}, \rho_{2}$ and $\rho_{3}=1: 10$. See Table for full configuration details

model is defined by it is own terms of sharing and interactions among the operators. The models represent the expected practical implementations of the next generation of cellular wireless networks were more spectrum blocks will be traded between operators. For each of the proposed models we have derived the blocking probability of the individual operators and spectrum utilization to quantify and analyze the benefits of the proposed models. The formulation of the models applies whether the operators adopts FDM, TDMA, W-CDMA or TDCDMA radio technologies. In addition, the models apply to the downlink as well as the uplink communications. The analysis provide a way to quantify the benefits to operators when they adopt spectrum sharing.

\section{REFERENCES}

[1] M. Matinmikko, H. Okkonen, M. Palola, S. Yrjola, P. Ahokangas, and M. Mustonen, "Spectrum sharing using licensed shared access: the concept and its workflow for lte-advanced networks," IEEE Wireless Communications, vol. 21, no. 2, pp. 72-79, 2014.

[2] A. Palaios, J. Riihijarvi, P. Mahonen, V. Atanasovski, L. Gavrilovska, P. Van Wesemael, A. Dejonghe, and P. Scheele, "Two days of spectrum use in europe," in 7th International ICST Conference on Cognitive Radio Oriented Wireless Networks and Communications (CROWNCOM), 2012, pp. 24-29.

[3] M. M. Buddhikot, P. Kolodzy, S. Miller, K. Ryan, and J. Evans, "Dimsumnet: new directions in wireless networking using coordinated dynamic spectrum," in Sixth IEEE International Symposium on a World of Wireless Mobile and Multimedia Networks. Ieee, 2005, pp. 78-85.

[4] OfCom, "Application of spectrum liberalisation and trading to the mobile sector - A further consultation," http://stakeholders.ofcom.org.uk/binaries/consultations/spectrumlib/anne xes/annex10.pdf, The Office of Communications, Tech. Rep., 2009.

[5] M. J. Marcus, "Unlicensed cognitive sharing of tv spectrum: The controversy at the federal communications commission," IEEE Communications Magazine, vol. 43, no. 5, pp. 24-25, 2005.

[6] I. Sugathapala, I. Kovacevic, B. Lorenzo, S. Glisic et al., "Quantifying benefits in a business portfolio for multi-operator spectrum sharing," IEEE Transactions on Wireless Communications, vol. 14, no. 12, pp. 6635-6649, 2015.

[7] P. D. Mankar, B. R. Sahu, G. Das, and S. Pathak, "Evaluation of blocking probability for downlink in poisson networks," IEEE Wireless Communications Letters, vol. 4, no. 6, pp. 625-628, 2015.

[8] O. Al-Khatib, W. Hardjawana, and B. Vucetic, "Wireless networks virtualisation: Traffic modeling and spectrum sharing," in IEEE International Conference on Communications. IEEE, 2015, pp. 5859-5864.

[9] N. Nathani, G. Manna, and S. Dorle, "Network architecture model of infrastructure based mobile cognitive radio system in licensed band with blocking probability assessment," in Future Technologies Conference. IEEE, 2016, pp. 231-236.

[10] D. T. C. Wong, A. T. Hoang, Y.-C. Liang, and F. P. S. Chin, "Complete sharing dynamic spectrum allocation for two cellular radio systems," in 2008 IEEE 19th International Symposium on Personal, Indoor and Mobile Radio Communications. IEEE, 2008, pp. 1-5.

[11] Y. Fang and Y. Zhang, "Call admission control schemes and performance analysis in wireless mobile networks," IEEE Transactions on vehicular technology, vol. 51, no. 2, pp. 371-382, 2002.

[12] H. Elbadawy, "Modeling and analysis for heterogeneous wireless networks by using of multi-dimensional markov models," in Computer and Communication Engineering, 2008. ICCCE 2008. International Conference on. IEEE, 2008, pp. 1116-1120.

[13] R. Abozariba, M. Asaduzzaman, and M. Patwary, "Radio resource sharing framework for cooperative multi-operator networks with dynamic overflow modelling," IEEE Transactions on Vehicular Technology, 2016, In press.

[14] D. Gross and C. M. Harris, Fundamentals of queueing theory. Wiley, 1998. 